Advanced Studies in Pure Mathematics 18-II, 1990

Kähler Metrics and Moduli Spaces

pp. $229-256$

\title{
Compact Ricci-Flat Kähler Manifolds
}

\author{
Ichiro Enoki
}

In this part, we survey general results on compact Kähler manifolds $M$ with $c_{1}(M)_{\mathbb{B}}=0$. According to the solution of the Calabi conjecture by Yau [Ya], such a compact Kähler manifold $M$ admits a unique Ricci flat Kähler metric with given Kähler class. Our main interests here are applications of the existence of Einstein-Kähler metrics to studies on topological or holomorphic structures of compact Kähler manifolds $M$ with $c_{1}(M)_{\mathbb{R}}=0$.

\section{Table of Contents}

$\S 1$. Bogomolov decomposition

$\S 2$. Deformation

$\S 3$. Symplectic Kähler manifolds

$\S 4$. Period map and Weil-Peterson metric

$\S 5$. Examples

\section{$\S 1$. Bogomolov decomposition}

There are three fundamental types of compact Kähler manifolds whose real first Chern classes vanish:

(1) complex tori $T$;

(2) symplectic Kähler manifolds, i.e., compact Kähler manifolds $X$ of even dimension $2 m$ which have a holomorphic 2 -form $\varphi$ with $\varphi^{m}$ nowhere vanishing on $X$ (such $\varphi$ is called holomorphic symplectic 2-form);

(3) special unitary Kähler manifolds, i.e., compact Kähler manifolds $Y$ of dimension $n \geq 3$ such that the canonical bundle of $Y$ is trivial but $H^{0}\left(Y, \Omega^{p}\right)=0$ for $0<p<n$.

Some examples of compact symplectic Kähler manifolds are given in Section 5. These three types are fundamental in the sense that the following holds: 
Theorem 1.1 (Bogomolov decomposition). Let $M$ be a compact Kähler manifold with $c_{1}(M)_{\mathbb{R}}=0$. Then a certain finite unramified covering space $M^{\prime}$ of $M$ decomposes holomorphically,

$$
M^{\prime} \cong T \times X_{1} \times \cdots \times X_{r} \times Y_{1} \times \cdots \times Y_{s},
$$

into a direct product of a complex torus $T$ and simply connected manifolds $X_{i}, Y_{j}$, where

- $X_{i}$ are symplectic Kähler manifolds with $h^{2,0}\left(X_{i}\right)=1$;

- $Y_{j}$ are special unitary Kähler manifolds.

Moreover this decomposition is unique up to order.

Because of the uniqueness of the decomposition, a simply connected symplectic Kähler manifold $X$ with $h^{2,0}(X)=1$ cannot decompose any more. Such a manifold $X$ is called irreducible symplectic Kähler manifold.

Corollary 1.2. a) The Albanese map of a compact Kähler manifold $M$ with $c_{1}(M)_{\mathbb{R}}=0$ is surjective; and

b) any surjective holomorphic map $f: M \rightarrow N$ between compact Kähler manifolds $M, N$ with $c_{1}(M)_{\mathbb{R}}=0, c_{1}(N)_{\mathbb{R}}=0$ induces a structure of holomorphic fiber bundle with finite structure group.

Calabi [Ca-1] noted that the existence of Ricci-flat Kähler metric on $M$ would imply Corollary 1.2 for the Albanese map. Bogomolov [Bo-1] proved Theorem 1.1 for simply connected $M$ without using the Ricciflat Kähler metrics. The following proof using the Ricci-flat metric was found independently by S. Kobayashi [Ko1] and Michelson [Mi-1][Mi-2].

We begin by quoting several facts:

Cheeger-Gromoll's splitting theorem [CG]. Let $M$ be a compact Riemannian manifold with non-positive Ricci curvature. Then the universal covering space of $M$ splits into Riemannian direct product of a flat Euclidean space and a compact simply connected manifold.

The second is the classification of holonomy groups. Let $(M, g)$ be a Riemannian manifold of dimension $m$. Then the restricted holonomy group $\Phi_{o}(x)$ at $x \in M$ is always isomorphic to a subgroup of $S O(m)$ with the standard action of $S O(m)$ to $T_{p} M$. We call $M$ irreducible if the action of the restricted holonomy group $\Phi_{o}(x)$ to $T_{x} M$ is irreducible. According to Berger [Ber], if $M$ is irreducible but not locally symmetric, then the restricted holonomy group $\Phi_{p}$ is isomorphic to one of the 
following subgroups of $S O(m)$ (the action to $T_{x} M$ is the standard one induced by that of $S O(m))$ :

$$
\begin{array}{llll}
S O(m) ; & U(n) \quad(m=2 n) ; & S U(n) \quad(m=2 n) ; \\
S p(r) \quad(m=4 r) ; & S p(1) \cdot S p(r) \quad(m=4 r) ; & & \\
\operatorname{Spin}(9) \quad(m=16) ; & \operatorname{Spin}(7) \quad(m=8) ; & G_{2} .
\end{array}
$$

Recall that Ricci flat locally symmetric spaces are flat. Moreover, if $(M, g)$ is a Ricci-flat Kähler manifold, then the restricted holonomy group is a subgroup of $S U(n)(\subset S O(m)), m=2 n$. Thus we have

Theorem 1.3. Let $M$ be a irreducible Ricci-flat Kähler manifold with $\operatorname{dim}_{\mathbb{C}} M=n$. Then the restricted holonomy group of $M$ is either $S U(n)$ or $S p(r), n=2 r$; the action to the tangent space is standard.

We need one more. Let $G=\Phi(x)$ be the holonomy group of $M$ at $x \in M$ and $\left(\bigwedge^{p} T_{x}^{*} M\right)^{G}$ the space of $(p, 0)$-forms at $x$ invariant under the action of $G$.

Proposition 1.4. Let $M$ be a compact Ricci-flat Kähler manifold with the holonomy group $G$ at $x \in M$. Then $H^{0}\left(M, \Omega^{p}\right) \cong\left(\bigwedge^{p} T_{x}^{*} M\right)^{G}$.

In fact, let $\xi$ be a holomorphic $p$-form on $M$. Let $\|\xi\|$ be the pointwise norm of $\xi$. We compute the laplacian of $\|\xi\|^{2}$. Then, since the Ricci curvature is zero, by the Bochner formula we have $\triangle\|\xi\|^{2}=\|\nabla \xi\|^{2}$, where $\nabla$ is the covariant derivative. Integrating the both hands sides yields $\nabla \xi=0$. Conversely any parallel $(p, 0)$-form is holomorphic since the $(0,1)$-part of the covariant derivative $\nabla$ is $\bar{\partial}$. Therefore the mapping $\xi \mapsto \xi(x)$ gives an isomorphism: $H^{0}\left(M, \Omega^{p}\right) \cong\left(\bigwedge^{p} T_{x}^{*} M\right)^{G}$.

Now we can give

Proof of Theorem 1.1. Let $M$ be a compact Kähler manifold with $c_{1}(M)_{\mathbb{R}}=0$. Then by Yau [Ya] $M$ has a Ricci-flat Kähler metric. Let $\tilde{M}$ be the universal covering of $M$ and $\tilde{M}=E \times \prod_{i} M_{i}$ the de Rham decomposition of $\tilde{M}$, where $E$ is the flat-part. Since $M$ is Kähler, this decomposition is holomorphic and the flat part $E$ is the complex Euclidean space. In view of the Cheeger-Gromoll splitting theorem cited above, the remaining part $\prod_{i} M_{i}$ is compact. Therefore, by the theorem of Bieberbach (see $[\mathrm{KB}]$ ), there is a finite unramified covering $M^{\prime} \cong$ $T \times \prod_{i} M_{i}$ of $M$, where $T$ is a complex torus covered by $E$. Note that the holonomy group of $M_{i}$ coincides with the restricted holonomy group since $M_{i}$ is simply connected. By the classification of restricted holonomy groups by Berger (Theorem 1.3) the holonomy group $G_{i}$ of 
$M_{i}$ is isomorphic to either $S U\left(m_{i}\right)$ or $S p\left(r_{i}\right), m_{i}=2 r_{i}$, where $m_{i}=$ $\operatorname{dim}_{\mathbb{C}} M_{i}$. According to Weyl [Weyl, Chap. VI],

$$
\operatorname{dim}_{\mathbb{C}}\left(\bigwedge^{p} T_{x}^{*} M_{i}\right)^{G_{i}}= \begin{cases}1, & \text { if } G_{i} \cong S p\left(r_{i}\right), m_{i}=2 r_{i} \text { and } p \text { is even; } \\ 0, & \text { if } G_{i} \cong S U\left(m_{i}\right) \text { and } 0<p<m_{i} .\end{cases}
$$

Thus by Proposition $1.4 M_{i}$ is either a symplectic Kähler manifold with $h^{2,0}\left(M_{i}\right)=1$ or a special unitary Kähler manifold according as $G_{i}$ is isomorphic to $S p\left(r_{i}\right), m_{i}=2 r_{i}$, or $S U\left(m_{i}\right)$.

Q.E.D.

\section{§. Deformation}

Let $M$ be a compact complex manifold and $\mathcal{M} \rightarrow S$ the Kuranishi family of $M$. We call $S$ the (local) universal deformation space of $M$. This space always exits as complex analytic space $[\mathrm{Ku}]$ but in general not smooth (even non-reduced).

Theorem 2.1 (Tian [Ti], Todorov [To-2]). Let $S$ be the universal deformation space of a compact Kähler manifold $M$ with $c_{1}(M)_{\mathbb{R}}=0$. Then $S$ is smooth and $\operatorname{dim} S=H^{1}(M, \Theta)$.

Bogomolov [Bo-2] proved this theorem for a symplectic compact Kähler manifold $M$. On a symplectic manifold the sheaf $\Theta$ of holomorphic vector fields is isomorphic to the sheaf $\Omega^{1}$ of holomorphic 1 -forms. He showed that any obstruction for deformation, which is an element of $H^{2}(M, \Theta)$, should vanish, by regarding it as an element of $H^{2}\left(M, \Omega^{1}\right)$ via the isomorphism above and then calculating integrals over 3-cycles. Fujiki [Fu-3] also gave a proof for symplectic Kähler manifold, using its hyperKähler structure (cf. Section 3). The proof we overview here is due to Tien and Todorov.

Let $M$ be a compact Kähler manifold of dimension $n$ and $T M$ its holomorphic tangent bundle. For a holomorphic vector bundle $E$ over $M$ let $A^{p, q}(E)$ denote the space of $E$-valued smooth $(p, q)$-forms, which is also understood to be the space of $E \otimes \wedge^{p} T^{*} M$-valued $(0, q)$-forms.

According to the deformation theory of Kodaira-Spencer (cf. [Kod]), each small deformation of $M$ corresponds to $\varphi \in A^{0,1}(T M)$ with the integrability condition

$$
\bar{\partial} \varphi+\frac{1}{2}[\varphi, \varphi]=0 .
$$

What Tien [Ti] and Todorov [To-2] proved in fact is the following

Theorem 2.1'. Let $M$ be a compact Ricci-flat Kähler manifold. Then for each $T M$-valued harmonic $(0,1)$-form $\varphi_{1}$ there exists a unique series $\varphi_{\mu} \in A^{0,1}(T M), \mu \geqq 1$ such that for $\mu \geqq 2$ 
a) $\bar{\partial} \varphi_{\mu}+\frac{1}{2} \sum_{\nu=1}^{\mu}\left[\varphi_{\nu}, \varphi_{\mu-\nu}\right]=0$

b) $\bar{\partial}^{*} \varphi_{\mu}=0$.

Then by the argument in [KNS] ([Kod], Chap. 5) the power series $\varphi(t)=$ $\sum \varphi_{\mu} t^{\mu}$ converges for sufficiently small $|t|$, satisfying the integrability condition above. This shows that $M$ can be deformed in the direction of any element of $H^{1}(M, \Theta)$ and hence the Kuranishi space of $M$ is an open subset of $H^{1}(M, \Theta)$.

Since $K_{M}^{*} \cong \bigwedge^{n} T M$, the contraction induces a holomorphic isomorphism

$$
\alpha_{r}: K_{M}^{*} \otimes \bigwedge^{n-r} T^{*} M \rightarrow \bigwedge^{r} T M
$$

where $\bigwedge^{0} T M$ is understood to be a trivial line bundle. This extends to

$$
\alpha_{r}: A^{n-r, q}\left(K_{M}^{*}\right) \rightarrow A^{0, q}\left(\bigwedge^{r} T M\right),
$$

commuting with $\bar{\partial}$. Via $\alpha_{1}$, the Lie bracket on $\bigoplus_{q \geq 0} A^{0, q}(T M)$ induces a Lie bracket [ , ] on $\bigoplus_{q \geq 0} A^{n-1, q}\left(K_{M}^{*}\right)$ :

$$
\left[\alpha_{1}(\xi), \alpha_{1}(\eta)\right]:=\alpha_{1}([\xi, \eta]) \quad \text { for } \quad \xi, \eta \in \bigoplus_{q \geqq 0} A^{0, q}(T M) .
$$

On the holomorphic tensor bundle $E=\bigwedge^{r} T M \otimes \bigwedge^{s} T^{*} M$, the LeviCivita connection $\nabla$ of $M$ defines the hermitian connection relative to the induced metric and the exterior covariant derivative

$$
d^{\nabla}: \bigoplus_{p+q=k} A^{p, q}(E) \rightarrow \bigoplus_{p+q=k+1} A^{p, q}(E)
$$

Let $\partial$ be the $(1,0)$-component of $d^{\nabla}$.

Lemma 2.2. For $\xi, \eta \in A^{n-1,1}\left(K_{M}^{*}\right)$

$$
[\xi, \eta]=\partial\left(\beta\left(\alpha_{1}(\xi) \wedge \eta\right)\right)-\alpha_{0}(\partial \xi) \wedge \eta+\xi \wedge \alpha_{0}(\partial \eta)
$$

where $\beta$ is induced from an interior product

$$
T M \otimes \bigwedge^{n-1} T^{*} M \rightarrow \bigwedge^{n-2} T^{*} M
$$

Proof. Recall that $K_{M}$ and $K_{M}^{*}$ are flat line bundles with the induced metrics. Relative to a parallel local trivialization $\omega$ of $K_{M}$, define $\operatorname{div}_{\omega} X$ for a local holomorphic vector field $X$ by

$$
\left(\operatorname{div}_{\omega} X\right) \omega=\mathcal{L}_{X} \omega
$$


where $\mathcal{L}_{X}$ is the Lie derivative with respect to $X$. Let $\left.Z\right\lrcorner \omega$ denote the interior product of $\omega$ with a vector field $Z$. Then $\partial \circ \beta$ corresponds to $\operatorname{div}_{\omega}$ and the lemma reduces to the fundamental properties of Lie derivative: for holomorphic vector fields $X, Y$ we have

$$
\begin{aligned}
\left.\mathcal{L}_{X}(Y\lrcorner \omega\right) & \left.\left.=\left(\operatorname{div}_{\omega} X\right) Y\right\lrcorner \omega+\left(\mathcal{L}_{X} Y\right)\right\lrcorner \omega & & \text { (the derivation rule) } \\
& =\partial(X\lrcorner Y\lrcorner \omega)+X\lrcorner \partial(Y\lrcorner \omega) & & \text { (H. Cartan's formula) }
\end{aligned}
$$

and $\mathcal{L}_{X} Y=[X, Y]$.

Q.E.D.

Since $K_{M}^{*}$ is a flat line bundle and $M$ is Kähler, the Hodge theory holds also for $K_{M}^{*}$-valued $(p, q)$-forms; in particular every $K_{M}^{*}$-valued $\bar{\partial}$-harmonic form is $\partial$-closed and the $\partial \bar{\partial}$-lemma holds:

Lemma 2.3 (cf. $[\mathrm{GH}]$ p. 149). If $K_{M}^{*}$-valued $\bar{\partial}$-closed $(p, q)$-form $\eta$ is $\partial$-exact, then there exists $a(p-1, q-1)$-form $\gamma$ such that $\eta=\bar{\partial} \partial \gamma$ and $\bar{\partial}^{*} \bar{\partial} \gamma=0$.

The flatness of $K_{M}$ also implies the following

Lemma 2.4. $\bar{\partial}^{*} \alpha_{1}(\xi)=\alpha_{1} \bar{\partial}^{*} \xi$ for $\xi \in A^{n-1,1}\left(K_{M}^{*}\right)$.

In fact, the inverse of $a^{(1)}$ can be obtained by contracting with $\omega$ and then tensoring $\omega^{*}$, where $\omega$ is a parallel local trivialization of $K_{M}$ and $\omega^{*}$ its dual. Express $\bar{\partial}^{*}$ using $\nabla$. Then, the lemma follows immediately since contractions and the covariant derivative commute each other.

Now the power series $\sum_{\mu \geqq 1} \varphi_{\mu} t^{\mu}$ in the theorem can be constructed inductively in terms of $\xi_{\mu} \in \bar{A}^{n-1,1}\left(K_{M}^{*}\right)$ with $\varphi_{m}=\alpha_{1}\left(\xi_{\mu}\right)$. The conditions a), b) correspond to
a) $\bar{\partial} \xi_{\mu}+\frac{1}{2} \sum_{\nu=1}^{\mu}\left[\xi_{\nu}, \xi_{\mu-\nu}\right]=0$,
b) $)^{\prime} \quad \bar{\partial}^{*} \xi_{\mu}=0$.

We pose moreover

c) $)^{\prime} \partial \xi_{\mu}=0$.

Since $\varphi_{1}$ and hence $\xi_{1}$ are harmonic, $\xi_{1}$ satisfies the conditions above by Lemma 2.4. Suppose there are determined $\xi_{1}, \ldots, \xi_{\mu}$ satisfying a)', b) $)^{\prime}$ and c $)^{\prime}$. Then each $\left[\xi_{\nu}, \xi_{\mu-\nu}\right]$ is $\partial$-exact by c) ${ }^{\prime}$ and Lemma 2.2 ; the sum is $\bar{\partial}$-closed by condition a $)^{\prime}$. Hence, by the $\partial \bar{\partial}$-lemma, Lemma 2.4 , there exists $\xi_{\mu+1}$ satisfying a) $\left.)^{\prime}, \mathrm{b}\right)^{\prime}$ and $\left.\mathrm{c}\right)^{\prime}$. By condition b) this series is unique. 


\section{$\S 3 . \quad$ Symplectic manifolds}

In this section we discuss the structure of de Rham cohomology ring of a symplectic Kähler manifold. We begin by recalling Kähler case briefly.

Kähler Case. Let $M$ be a compact Kähler manifold of dimension $n$. We first note that any parallel endmorphism $\theta$ of the tangent bundle $T M$ of $M$ induces a derivation of the de Rham cohomology ring $H^{*}(M, \mathbb{R})$ of $M$. In fact we have ([Li-1] or see [Li-2])

Proposition 3.1. Let $M$ be a Riemannian manifold and $\triangle$ the Laplacian on $p$-forms. If $h$ is a parallel endmorphism of $\wedge^{p} T^{*} M$, then $\triangle(h \alpha)=h \triangle \alpha$ for any $p$-form $\alpha$.

The complex structure $J$ on a Kähler manifold $M$ is a parallel endmorphism of $T M$. Let $v(J)$ denote the derivation (over $\mathbb{R}$ ) on $\wedge^{*} T^{*} M$ induced by $J$, namely, $v(J) \varphi=\sqrt{-1}(p-q) \varphi$ for $(p, q)$-form $\varphi$. Recall that $T M$ admits another parallel endmorphism, that is, the identity id; and it induces a derivation $v$ (id) given by $v($ id $) \varphi=(p+q) \varphi$ for $(p, q)$ form $\varphi$. These two derivations generate a Lie algebra corresponding to a Lie group $U(1) \times \mathbb{R}^{*} \cong \mathbb{C}^{*}$. Thus we have

Proposition 3.2 (see [We]). Let $M$ be a compact Kähler manifold. Then there is a real representation $\rho$ of $\mathbb{C}^{*} \cong U(1) \times \mathbb{R}^{*}$ to the algebra automorphism group of the cohomology ring $H^{*}(M, \mathbb{R})$.

Let $H^{p, q}(M) \subset H^{p+q}(M, \mathbb{C})$ be a subspace spanned by classes of $d$ closed $(p, q)$-forms in the de Rham cohomology group of $M$. Then the Hodge decomposition,

$$
H^{k}(M, \mathbb{C}) \cong \bigoplus_{p+q=k} H^{p, q}(M), \quad \bar{H}^{p, q}(M)=H^{q, p}(M),
$$

is nothing but the decomposition of $H^{*}(M, \mathbb{C}) \cong H^{*}(M, \mathbb{R}) \otimes \mathbb{C}$ into the isotypical components of the real representation $\rho$ in Proposition 3.2 above (an isotypical component is, by definition, a direct sum of one and the same irreducible representation). Moreover $\rho$ does not depend on a Kähler metric of $M$. In fact, for $z=\exp (s+\sqrt{-1} \theta), s, \theta \in \mathbb{R}$, we have

$$
\rho(z)=\sum_{p, q} \exp ((p+q) s+\sqrt{-1}(p-q) \theta) \pi^{p, q},
$$

where $\pi^{p, q}: H^{r}(M, \mathbb{C}) \rightarrow H^{p, q}(M)$ denote the projection. 
Let $\omega$ be a Kähler form on $M$. Let $L$ be the multiplication operator on $H^{*}(M, \mathbb{C})$ by the Kähler class of $\omega$ and $\Lambda$ the adjoint operator of $L$. For $k \leq n, n=\operatorname{dim} M$ we set

$$
\begin{aligned}
H^{k}(M, \mathbb{C})_{\omega} & =\left\{\alpha \in H^{k}(M, \mathbb{C}) \mid L^{n-k+1} \alpha=0\right\}, \\
H^{p, q}(M)_{\omega} & =H^{k}(M, \mathbb{C})_{\omega} \cap H^{p, q}(M) .
\end{aligned}
$$

Then the strong Lefschetz theorem says

$$
\begin{aligned}
& L^{n-k}: H^{n-k}(M, \mathbb{R}) \cong H^{n+k}(M, \mathbb{R}), \\
& H^{k}(M, \mathbb{R})=\bigoplus_{r \geq 0} L^{r} H^{k-2 r}(M, \mathbb{R})_{\omega} .
\end{aligned}
$$

Elements of $H^{k}(M, \mathbb{C})_{\omega}$ are called $\omega$-effective (or primitive).

hyperKähler manifold. Let $M$ be now a symplectic Kähler manifold of complex dimension $2 n$. According to the solution of the Calabi conjecture by Yau [Ya] there is a unique Ricci flat Kähler metric $g$ with given Kähler class. Then $(M, g)$ has a structure of hyperKähler manifold (for definition, see below). Before stating results on the structure of cohomology ring of $M$, we introduce first a few notions related to hyperKähler manifolds.

The restricted holonomy group of $(M, g)$ is a subgroup of $S p(n)$. (It is exactly $S p(n)$ if $M$ is irreducible.) Hence the ring of parallel endomorphisms of the tangent bundle $T M$ of $M$ contains a subalgebra $\dot{H}$ isomorphic to (and identified with) the standard quaternion algebra over $\mathbb{R}$. Let $P=\left\{\lambda \in \mathbb{H} \mid \lambda^{2}=-1\right\}$. Then each $\lambda$ defines an integrable complex structure on $M$ so that $g$ is a Kähler metric under this complex structure. Let $M_{\lambda}$ denote the manifold $M$ with complex structure defined by $\lambda$ and $\omega_{\lambda}$ the Kähler form of $g$ relative to $\lambda$. Thus we have a family $\left\{\left(M_{\lambda}, \omega_{\lambda}\right)\right\}_{\lambda \in P}$ of Kähler structures, which is called the Calabi family; and the manifold $M$ together with this family is called a hyperKähler manifold. Moreover the family $\left\{\omega_{\lambda}\right\}_{\lambda \in P}$ of $d$-closed 2-forms on $M$ defines a 3 -dimensional subspace $F \subset H^{2}(M, \mathbb{R})$, which we call the hyperKähler 3-space associated to $(M, g)$.

Each member $\left(M_{\lambda}, \omega_{\lambda}\right)$ of the Calabi family is again a symplectic Kähler manifold (as we will see a little later). Conversely a Kähler class $[\omega]$ on $M$ and a 1-dimensional subspace of $H^{0}\left(M, \Omega^{2}\right)$ spanned by a holomorphic symplectic 2-form $\varphi$ determine a hyperKähler structure on $M$. For a symplectic Kähler manifold $M$ with $h^{2,0}(M)=1$, in particular, a hyperKähler structure on $M$ is equivalent to a polarization on $M$, i.e., fixing a Kähler class on $M$. 
Let $H^{*}:=\mathbb{H}-\{0\}$. By Proposition 3.1 each parallel endomorphism of $T M$ induces an algebra automorphism of $H^{*}(M, \mathbb{R})$. Therefore, corresponding to Proposition 3.2 above we have

Proposition 3.3. Let $M$ be a hyperKähler manifold. Then there is a real representation $\rho_{\mathrm{HK}}$ of $H^{*} \cong S p(1) \times \mathbb{R}_{>0}$ to the algebra automorphism group of the cohomology ring $H^{*}(M, \mathbb{R})$.

The decomposition of $H^{k}(M, \mathbb{R})$ into isotypical components of the action of $\rho_{\mathrm{HK}}\left(\mathbb{H}^{*}\right)$ is compatible to the Hodge decomposition relative to any complex structure $\lambda \in P$. Note that, however, this decomposition depends on the hyperKähler structure of $M$.

Corollary 3.4 ([Wa]). Every odd dimensional Betti number of a compact hyperKähler manifold is divisible by 4 .

This follows from Proposition 3.3 and results on the representation of $H^{*}([\mathrm{Fu}-3])$. The following argument is taken from Wakakuwa's long forgotten paper [Wa].

Proof. Let $\xi, \eta$ be harmonic forms of odd degree on a compact hyperKähler manifold with Ricci flat Kähler metric g. Assume that $\xi$ is orthogonal to $\eta$ with respect to the $L^{2}$-inner product. Since $g$ is hermitian relative to the complex structure corresponding to each $\lambda \in P$, we have $g(\rho(\lambda) \xi, \rho(\lambda) \eta)=g(\xi, \eta)$. On the other hand $\rho(\lambda)^{2} \xi=-\xi$ for $\lambda \in P$ since the degree of $\xi$ is odd. Thus the de Rham classes of $\xi$ and $\rho(\lambda) \xi, \lambda \in P$, span a 4-dimensional subspace in the cohomology group and they are all orthogonal to $\eta$.

Q.E.D.

We examine here the action of $\rho_{\mathrm{HK}}\left(\mathbb{H}^{*}\right)$ more closely. For $\lambda \in P$ let $v(\lambda)$ denote the derivation on $H^{*}(M, \mathbb{C})$ induced by the complex structure $\lambda$. Then $v(\mu) w_{\lambda}=2 \omega_{\lambda \mu}$. Let $(\lambda, \mu, \nu)$ be a standard basis of pure quaternions such that

$$
\begin{gathered}
l^{2}=\mu^{2}=\nu^{2}=-1 \\
l \mu=-\mu \lambda=\nu, \mu \nu=-\nu \mu=\lambda, \nu \lambda=-\lambda \nu=\mu .
\end{gathered}
$$

Then $v(\lambda) \alpha=\sqrt{-1}(p-q) \alpha$ for $\alpha \in H^{p, q}(M)$, relative to the complex structure $\lambda$, and

$$
\begin{aligned}
& (v(\mu)+\sqrt{-1} v(\nu))\left(H^{p, q}(M)\right) \subset H^{p+1, q-1}(M), \\
& (v(\mu)-\sqrt{-1} v(\nu))\left(H^{p, q}(M)\right) \subset H^{p-1, q+1}(M) .
\end{aligned}
$$


Moreover

$$
\varphi_{\lambda}:=\omega_{\mu}+\sqrt{-1} \omega_{\nu}=\sqrt{-1}(v(\mu)+\sqrt{-1} v(\nu)) \omega_{\lambda}
$$

is a holomorphic symplectic 2 -form under the complex structure $\lambda$. The hyperKähler 3 -space $F$, spanned by the de Rham cohomology classes of $\omega_{\lambda}, \omega_{\mu}$ and $\omega_{\nu}$, is identified with the space of pure quaternions, i.e., the Lie algebra $s p(1)$. Thus we have

Proposition 3.6. For a compact hyperKähler manifold $M$,

1) $\rho_{\mathrm{HK}}\left(\mathbb{H}^{*}\right) \cong \mathbb{H}^{*} \cong S p(1) \times \mathbb{R}_{>0}$;

2) the hyperKähler 3 -space $F$ is stable under the action of $\rho_{\mathrm{HK}}\left(\mathbb{H}^{*}\right)$ and the action of $S p(1)$ on $F \cong s p(1)$ is identified with the adjoint action.

For each $\lambda \in P$ let $L_{\lambda}$ be the endomorphism of $H^{*}(M, \mathbb{R})$ defined by the multiplication with $\omega_{\lambda}$. For $k \leq 2 n$, define

$$
H^{k}(M, \mathbb{R})_{F}:=\left\{\alpha \in H^{k}(M, \mathbb{R}) \mid L_{\lambda}^{2 n-k+1} \alpha=0 \quad \text { for } \lambda \in P\right\} .
$$

An element of $H^{k}(M, \mathbb{R})_{F}$ called universally effective. Since each element $\omega_{\lambda}$ of the hyperKähler 3 -space $F$ is a Kähler form relative to the complex structure corresponding to $\lambda$, the strong Lefschetz theorem holds with respect to each $L_{\lambda}$. Moreover we have

Theorem 3.7 [Fu-3]. Let $M$ be a compact symplectic manifold with $\operatorname{dim}_{\mathbb{C}} M=4 n$. Let $N^{*}$ be the subalgebra of $H^{*}(M, \mathbb{R})$ generated by the hyperKähler 3-space $F$. Then:

1) The submodule $H_{\epsilon}^{*}(M, \mathbb{R})$ generates $H^{*}(M, \mathbb{R})$ as $N^{*}$-module and we have a natural direct sum decomposition

$$
H^{l}(M, \mathbb{R})=\bigoplus N^{l-k} H^{k}(M, \mathbb{R})_{F}
$$

2) If $l \leq n$, then the natural map

$$
N^{l-k} \otimes_{\mathbb{R}} H^{k}(M, \mathbb{R})_{F} \rightarrow N^{l-k} H^{k}(M, \mathbb{R})_{F}
$$

is an isomorphism of $\mathbb{H}^{*}$-module.

Let $\varphi$ be the holomorphic symplectic 2 -form on $M$. Let

$$
\begin{aligned}
& L_{\varphi}: H^{q}\left(M, \Omega^{p}\right) \rightarrow H^{q}\left(M, \Omega^{p+2}\right), \\
& L_{\bar{\varphi}}: H^{q}\left(M, \Omega^{p}\right) \rightarrow H^{q+2}\left(M, \Omega^{p}\right)
\end{aligned}
$$


be the linear maps defined by the multiplication with $\varphi$ and $\bar{\varphi}$ respectively. For $\gamma=\varphi$ or $\bar{\varphi}$ the space of $\gamma$-effective Dolbeault classes is defined by

$$
H^{q}\left(M, \Omega^{p}\right)_{\gamma}:=\left\{\alpha \in H^{q}\left(M, \Omega^{p}\right) \mid L_{\gamma}^{n-s+1} \alpha=0\right\},
$$

where $s=p$ or $q$ according to $\gamma=\varphi$ or $\bar{\varphi}$.

Theorem 3.8 [Fu-3]. Let $M$ be a compact symplectic Kähler manifold with $\operatorname{dim}_{\mathbb{C}} M=4 n$. Then:

1) The linear maps

$$
\begin{gathered}
L_{\varphi}^{n-p}: H^{q}\left(M, \Omega^{p}\right) \rightarrow H^{q}\left(M, \Omega^{2 n-p}\right) \quad \text { for } p<n, \text { and } \\
L_{\bar{\varphi}}^{n-q}: H^{q}\left(M, \Omega^{p}\right) \rightarrow H^{2 n-q}\left(M, \Omega^{p}\right) \quad \text { for } q<n,
\end{gathered}
$$

are both isomorphic.

2) For any $p, q \geq 0$ we have the direct sum decopositions

$$
\begin{gathered}
H^{q}\left(M, \Omega^{p}\right)=\bigoplus_{r \geq n-p} L_{\varphi}^{r} H^{q}\left(M, \Omega^{p}\right)_{\varphi}, \\
H^{q}\left(M, \Omega^{p}\right)=\bigoplus_{r \geq n-q} L_{\bar{\varphi}}^{r} H^{q-2 r}\left(M, \Omega^{p}\right)_{\bar{\varphi}} .
\end{gathered}
$$

Theorems 3.7 and 3.8 are, respectively, hyperKähler and holomorphic symplectic versions of the strong Lefschetz theorem for Kähler manifolds. Recall that the strong Lefschetz theorem is a consequence of the fact that the de Rham cohomology ring of a compact Kähler manifold admits an $s l(2)$-action generated by the operator $L$ and its formal adjoint $\Lambda$. One can proof Theorem 3.8 similarly by considering an $s l(2)$-action on $\bigoplus_{p} H^{q}\left(M, \Omega^{p}\right)$ (or $\bigoplus_{q} H^{q}\left(M, \Omega^{p}\right)$ ) generated by $L_{\varphi}$ (or $L_{\bar{\varphi}}$ ) and its formal adjoint. We shall give here a proof of Theorem 3.7, which uses the fact that $L_{\lambda}$, their formal adjoint operators, and $v(\lambda), \lambda \in P$, generate an $s p(2)$-action on the de Rham cohomology ring.

Proof of Theorem 3.7. We fix a Ricci-flat Kähler structure on $M$. Define an operator $H$ by $H \varphi=(p+q-2 n) \varphi$ for $(p, q)$-form $\varphi$. The complex structure corresponding to $\lambda \in P$ induces a derivation $v(\lambda)$ (over $\mathbb{R}$ ) of the space of forms. Moreover, for $\lambda \in P$, let $L_{\lambda}$ denote the multiplication by $\omega_{\lambda}$ and let $\Lambda_{\lambda}$ be its formal adjoint. Then these operators act on the space of harmonic forms by Proposition 3.1. We shall determine the commutator relations.

First of all, for each $\lambda \in P$ we already know that $H, L_{\lambda}$ and $\Lambda_{\lambda}$ generate $s l(2)$ :

$$
\left[H, L_{\lambda}\right]=-2 L_{\lambda},\left[H, \Lambda_{\lambda}\right]=2 \Lambda_{\lambda},\left[L_{\lambda}, \Lambda_{\lambda}\right]=H,
$$


and $v(\lambda), \lambda \in P$, generate $s p(1)$ :

$$
[v(\lambda), v(\mu)]=-2 v(\lambda \mu) \text { for } \lambda, \mu \in P .
$$

To derive other relations, take a standard basis of the pure quaternions, say $\lambda, \mu$ and $\nu$, so that $\lambda$ corresponding to the fixed complex structure. Then $\varphi:=(1 / 2)\left(\omega_{\mu}+\sqrt{-1} \omega_{\nu}\right)$ is a holomorphic symplectic 2 -form. Let $L_{\varphi}$ denote the multiplication by $\varphi$ and $\Lambda_{\varphi}$ its formal adjoint. Moreover let $L_{\bar{\varphi}}$ and $\Lambda_{\bar{\varphi}}$ denote the complex conjugate of $L_{\varphi}$ and $\Lambda_{\varphi}$ respectively. Since $v(\lambda) \alpha=\sqrt{-1}(p-q) \alpha$ and $\varphi$ is of type $(2,0)$, we have $\left[v(\lambda), L_{\varphi}\right]=$ $2 \sqrt{-1} L_{\varphi}$. Taking the real part and its adjoint, we obtain

$$
\left[v(\lambda), L_{\mu}\right]=-2 L_{\nu}, \quad\left[v(\lambda), \Lambda_{\mu}\right]=2 \Lambda_{\nu}
$$

Let $\alpha$ be a $(p, q)$-form. By the same calculation as in the Kähler case we have

$$
\left[L_{\varphi}, \Lambda_{\bar{\varphi}}\right]=0, \quad\left[L_{\varphi}, \Lambda_{\varphi}\right] \alpha=(p-n) \alpha .
$$

It follows

$$
\begin{aligned}
\sqrt{-1}\left[L_{\mu}, \Lambda_{\nu}\right] \alpha & =\left[L_{\varphi}+L_{\bar{\varphi}}, \Lambda_{\varphi}-\Lambda_{\bar{\varphi}}\right] \alpha \\
& =(p-q) \alpha=\frac{1}{\sqrt{-1}} v(\lambda) \alpha .
\end{aligned}
$$

Consequently, if $\lambda \neq \mu$, then

$$
\begin{gathered}
{\left[v(\lambda), L_{\mu}\right]=-2 L_{\lambda \mu}, \quad\left[v(\lambda), \Lambda_{\mu}\right]=2 \Lambda_{\lambda \mu}} \\
{\left[L_{\lambda}, \Lambda_{\mu}\right]=-v(\lambda \mu) .}
\end{gathered}
$$

Any other commutator of $H, L_{\lambda}, \Lambda_{\lambda}, v(\lambda), \lambda \in P$, which does not appear in $(*),(* *)$ or $(* * *)$ is zero.

Let $\mathcal{H}^{*}$ be the space of harmonic forms on $M$ with coefficients in $\mathbb{C}$. By the above commutator relations, the complex Lie algebra generated by $H, v(\lambda), L_{\lambda}$ and $\Lambda_{\lambda}, \lambda \in P$, is isomorphic to $s p(2, \mathbb{C})$. Since $s p(2, \mathbb{C})$ is semi-simple, $\mathcal{H}^{*}$ is decomposed into a direct sum of irreducible $s p(2, \mathbb{C})$ submodules. Let $V \subset \mathcal{H}^{*}$ be an irreducible subspace. For a subspace $\mathfrak{h} \subset \operatorname{sp}(2, \mathbb{C})$ and $U \subset V$ we denote:

$$
\mathfrak{h} U:=\left\{X_{1} X_{2} \cdots X_{m} \gamma \mid X_{i} \in \mathfrak{h}, \gamma \in U\right\} .
$$

For $\lambda \in P$ let $\mathfrak{g}_{\lambda}$ be the Lie subalgebra generated by $H, L_{\lambda}$ and $\Lambda_{\lambda}$. Let $V_{\lambda} \subset V$ be a $\mathfrak{g}_{\lambda}$-irreducible subspace. As a $\mathfrak{g}_{\lambda}$-module, $V_{\lambda}$ is generated by an $\omega_{\lambda}$-effective element $v_{\lambda} \in V_{\lambda}$. Choose $\lambda \in P$ so that the degree as a form of $v_{\lambda}$ is minimal among those of $v_{\mu}, \mu \in P$. Then, since $\mathfrak{g}_{\mu} v_{\lambda}$ is 
$\mathfrak{g}_{\mu}$-irreducible, $v_{\lambda}$ is $\omega_{\mu}$-effective for any $\mu \in P$, i.e., universally effective. Let $\mathcal{L}$ be the subspace spanned by $L_{\lambda}, \lambda \in P$ and $\mathcal{D}$ be the subspace spanned by $v(\lambda), \lambda \in P$. It follows by $(*),(* *)$ and $(* * *)$ that $\mathcal{L} \mathcal{D} v_{\lambda}$ is stable under the action of $s p(2, \mathbb{C})$ and hence $V=\mathcal{L} \mathcal{D} v_{\lambda}$. Moreover by $(* * *)$ each element of $\mathcal{D} v_{\lambda}$ is universally effective and of the same degree. This proves 1 ) of the theorem.

To prove 2) of the theorem, we recall the representations of $s p(2, \mathbb{C})$ (cf. [Weyl]). For any pair $\left(f_{1}, f_{2}\right)$ of integers with

$$
f_{1} \geq f_{2} \geq 0, \quad f_{1}+f_{2}=d \geq 1
$$

there is an irreducible representation $V\left(f_{1}, f_{2}\right)$ of $s p(2, \mathbb{C})$ and any irreducible representation is equivalent to $V\left(f_{1}, f_{2}\right)$ for some $\left(f_{1}, f_{2}\right)$. Moreover $V\left(f_{1}, f_{2}\right)$ and $V\left(g_{1}, g_{2}\right)$ are equivalent if and only if $\left(f_{1}, f_{2}\right)=$ $\left(g_{1}, g_{2}\right)$.

We use the following characterization of $V\left(f_{1}, f_{2}\right)$. Let $V$ be an irreducible representation of $s p(2, \mathbb{C})$. Consider the set $E$ consisting of all pairs $(r, s)$ of eigen values $r$ of $H$ and eigen values $s$ of $v(\lambda)$ on $V$. Let $\left(r_{0}, s_{0}\right)$ be the maximal element of $E$ with respect to the lexicographical order. Then $V$ is equivalent to $V\left(f_{1}, f_{2}\right)$ with $f_{1}+f_{2}=r_{0}$ and $f_{1}-f_{2}=$ $s_{0}$.

To observe the action of $s p(2, \mathbb{C})$ on $V\left(f_{1}, f_{2}\right)$, we shall realize it in an exterior algebra. Let $V_{0}$ be a complex vector space with basis $z_{1}, \ldots, z_{n}, w_{1}, \ldots, w_{n}$. In $V:=\bigoplus_{p, q \geq 0} \wedge^{p} V_{0} \wedge \wedge^{q} \bar{V}_{0}$, we set

$$
\omega_{V}=\sum_{i=1}^{n}\left(z_{i} \wedge \bar{z}_{i}+w_{i} \wedge \bar{w}_{i}\right), \quad \varphi_{V}=\sum_{i=1}^{n} z_{i} \wedge w_{i}
$$

Let $L_{\omega_{V}}, L_{\varphi_{V}}$ and $L_{\bar{\varphi}_{V}}$ denote the multiplication in $V$ by $\omega_{V}, \varphi_{V}$ and $\bar{\varphi}_{V}$ respectively. Moreover let $\Lambda_{\omega_{V}}, \Lambda_{\varphi_{V}}$ and $\Lambda_{\bar{\varphi}_{V}}$ be the adjoint operators of $L_{\omega_{V}}, L_{\varphi_{V}}$ and $L_{\bar{\varphi}_{V}}$ respectively with respect to the hermitian metric defined by $\omega_{V}$. Then these generate the action of $s p(2, \mathbb{C})$ on $V$. Since these operators just correspond to $L_{\omega}, L_{\varphi}, L_{\bar{\varphi}}, \Lambda_{\omega}, \Lambda_{\varphi}$ and $\Lambda_{\bar{\varphi}}$ respectively, we will use the same symbols as before. Set

$$
X:=\left[\Lambda_{\omega}, L_{\bar{\varphi}}\right], \quad Y:=\left[\Lambda_{\omega}, L_{\varphi}\right] .
$$

Choose integers $0 \leq q \leq p \leq n$ so that $f_{1}=n-q$ and $f_{2}=n-p$. Let $\xi:=z_{1} \wedge \cdots \wedge z_{p} \wedge \bar{w}_{1} \wedge \cdots \wedge \bar{w}_{q}$. Then $s p(2, \mathbb{C}) \xi$ is equivalent to $V\left(f_{1}, f_{2}\right)$. In fact we have

$$
\begin{gathered}
\Lambda_{\omega} \xi=\Lambda_{\varphi} \xi=\Lambda_{\bar{\varphi}} \xi=Y \xi=0 \\
H \xi=(2 n-(p+q)) \xi, \quad v(\lambda) \xi=(p-q) \xi .
\end{gathered}
$$


Therefore the eigen space of $H$ with the maximal eigen value, $2 n-(p+q)$, in $s p(2, \mathbb{C}) \xi$ is spanned by $X^{r} \xi, 0 \leq r \leq p-q$. Moreover $v(\lambda) X^{r} \xi=$ $(p-q-r) X^{r} \xi$. Note that all $X^{r} \xi$ are universally effective.

Now we complete the proof of 2). Let $\xi(a, b, c, r)=L_{\omega}^{a} L_{\varphi}^{b} L_{\bar{\varphi}}^{c} X^{r} \xi$. Then, among these elements, $\xi(a, b, c, r)$ is characterized by the fact that it contains the term $\Xi(k) \wedge \Omega(a) \wedge \Phi(b) \wedge \Psi(c)$, where

$$
\begin{gathered}
\Xi(r)=\bigwedge_{i=1}^{p-r} z_{i} \wedge \bigwedge_{i=1}^{q} \bar{w}_{i} \wedge \bigwedge_{j=p-r+1}^{p} \bar{w}_{j}, \quad \Omega(a)=\bigwedge_{j=p+1}^{p+a}\left(z_{j} \wedge \bar{z}_{j}\right) \\
\Phi(b)=\bigwedge_{k=p+a+1}^{p+a+b}\left(z_{k} \wedge w_{k}\right), \quad \Psi(c)=\bigwedge_{k=p+a+b+1}^{p+a+b+c}\left(\bar{z}_{k} \wedge \bar{w}_{k}\right) .
\end{gathered}
$$

Therefore $\xi(a, b, c, r), 2 a+2 b+2 c+p+q \leq n, k \leq p-q$, are linearly independent.

Q.E.D.

Theorem 3.9 ([Bea-2], [Fu-3]). Let $M$ be a compact symplectic Kähler manifold with $h^{2,0}(M)=1$. Let $v(\alpha):=\int_{M} \alpha^{2 n}$ for $\alpha \in$ $H^{2}(M, \mathbb{R})$. Then there is a unique quadratic form $f$ on $H^{2}(M, \mathbb{R})$ such that

(1) $f$ is non-degenerate with signature $\left(3, b_{2}(M)-3\right) ; f(\gamma)>0$ for any Kähler class $\gamma$;

(2) $f(\alpha)^{n}=v(\alpha)$ for $\alpha \in H^{2}(M, \mathbb{R})$;

(3) for $\alpha, \beta \in H^{2}(M, \mathbb{R})$

$$
\begin{aligned}
& v(\alpha)^{2} f(\beta) \\
= & f(\alpha)\left[(2 n-1) v(\alpha) \int_{M} \alpha^{2 n-2} \beta^{2}-(2 n-2)\left(\int_{M} \alpha^{2 n-1} \beta\right)^{2}\right]
\end{aligned}
$$

(4) $f$ is $\mathbb{Q}$-valued on $H^{2}(M, \mathbb{Q})$.

We note that (2) of the theorem is due to Fujiki [Fu-3] and (3) is due to Beauville [Bea-2]. Both of their proofs use the Bogomolov unobstructed theorem for deformations; this unobstructed theorem can be proved using the existence of Ricci-flat metrics (see Section 2). The following proof of Theorem 3.8 uses the solution of the Calabi conjecture more directly.

Proof of Theorem 3.9. Let $M$ be a compact symplectic Kähler manifold with $h^{2,0}=1$. Let $\varphi$ be a symplectic holomorphic 2 -form on $M$ 
normalized so that $\int_{M}(\varphi \bar{\varphi})^{n}=1$. Following [Bea-2], for $\alpha \in H^{2}(M, \mathbb{R})$ let

$$
f_{o}(\alpha):=\frac{n}{2} \int_{M}(\varphi \bar{\varphi})^{n-1} \alpha^{2}+(1-n) \int_{M} \varphi^{n-1} \bar{\varphi}^{n} \alpha \cdot \int_{M} \varphi^{n} \bar{\varphi}^{n-1} \alpha .
$$

We shall show that $f_{o}$ multiplied by a suitable positive constant has desired properties.

Since $f_{o}$ is a polynomial on $H^{2}(M, \mathbb{R})$, it suffices to consider on an open subset of $H^{2}(M, \mathbb{R})$. Therefore we may assume that the $(1,1)$ component $\alpha^{(1.1)}$ of $\alpha \in H^{2}(M, \mathbb{R})$ is a Kähler class. According to the solution of the Calabi conjecture by Yau [Ya], there is a Ricci-flat Kähler metric $g$ on $M$ with Kähler class $\alpha^{(1,1)}$. Let $\omega$ be the Kähler form of $g$. We may assume $\int_{M} \omega^{2 n}=1$. In the following we consider this Riemannian structure. Then the symplectic form $\varphi$ on $T_{p} M, p \in M$, is invariant under the action of the holonomy group, $S p(4 n)$ with the standard action. Hence $\varphi$ can be written as

$$
\varphi=\frac{(2 n) !}{2^{2 n}(n !)^{2}} \sum_{i=1}^{n} u_{i} \wedge v_{i}
$$

with a suitable unitary basis $u_{1}, \ldots, u_{n}, v_{1}, \ldots, v_{n}$ of $T_{p}^{*} M$; note that the Kähler form $\omega$ at $p$ is given by

$$
\omega=\frac{\sqrt{-1}}{2} \sum_{i=1}^{n}\left(u_{i} \wedge \bar{u}_{i}+v_{i} \wedge \bar{v}_{i}\right)
$$

Since $\varphi$ and $\omega$ are parallel, it follows that $(\varphi \bar{\varphi})^{n-1} \omega^{2}=c \omega^{2 n}$ on the whole $M$, where $c$ is a positive constant depending only on the dimension of $M$. Hence $f_{o}(\alpha)=c^{\prime} \int_{M} \alpha^{2 n}$ by a direct calculation.

To prove (3) we shall use the $S p(1)$-action on the cohomology ring $H^{*}(M, \mathbb{R})$. First we show that $f_{o}$ is invariant under this action. By Theorem 3.7 we have

$$
H^{2}(M, \mathbb{R})=F \oplus H^{2}(M, \mathbb{R})_{F},
$$

as $S p(1)$-module, where $F$ is the hyperKähler 3 -space. Since $h^{2,0}(M)=$ 1 , any element of $H^{2}(M, \mathbb{R})_{F}$ is of type $(1,1)$. Therefore the action of $S p(1)$ on $H^{2}(M, \mathbb{R})_{F}$ is trivial by (3.5). Since $\omega_{\lambda}^{2 n}, \lambda \in P$, are the volume form of the same metric, $f_{o}\left(\omega_{\lambda}\right), \lambda \in P$, are all equal by (2). By (2) of Proposition 3.6 it follows that $f_{0}$ is $S p(1)$-invariant.

Since the action of $S p(1)$ on $H^{4 n}(M, \mathbb{R})$ is trivial, both hand sides of $(3)$ is $S p(1)$-invariant. Moreover $S p(1)$ acts transitively on the hyperKähler 3 -space $F$ by Proposition 3.6. Therefore we may assume that 
$\alpha$ is of type $(1,1)$ and hence $\alpha=\omega$. Let $\beta_{o}$ be the universally effective part of $\beta \in H^{2}(M, \mathbb{R})$ so that $\beta$ can be written as $\beta=\omega+c \varphi+\bar{c} \bar{\varphi}+\beta_{o}$ for some $c \in \mathbb{C}$. Note that

$$
\omega_{\lambda}^{a} \omega_{\mu}^{b} \omega_{\nu}^{c} \beta_{o}=0 \quad \text { for } a+b+c=2 n-1, a, b, c \geq 0 .
$$

Therefore $\omega^{2 n-2} \varphi \beta_{0}=0$ and $(\varphi \wedge \bar{\varphi})^{n-1} \varphi \beta_{o}=0$. Now we have (3) by a direct calculation.

In particular $f_{o}$ is positive definite on the hyperKähler 3-space $F$. By Hodge bilinear relation $\int_{M} \omega^{2 n-2} \gamma^{2}<0$ for $\gamma \in H^{2}(M, \mathbb{R})_{F}$. Hence by $(3) f_{o}(\gamma)<0$ for $\gamma \in H^{2}(M, \mathbb{R})_{F}$. Thus the signature of $f_{o}$ is $\left(3, b_{2}(M)-3\right)$.

Since $f_{o}(\varphi+\bar{\varphi}), v(\varphi+\bar{\varphi})>0$, we can choose $\gamma \in H^{2}(M, \mathbb{Q})$ sufficiently near to $\varphi+\bar{\varphi}$ so that $f_{o}(\gamma)>0, v(\gamma)>0$. Note that $v(\gamma)$ is a rational number. Thus by $(3) f:=f_{o}(\gamma)^{-1} f_{o}$ is $\mathbb{Q}$-valued on $H^{2}(M, \mathbb{Q})$.

Q.E.D.

Thus $H^{2}(X, \mathbb{Q})$ of a symplectic Kähler manifold $X$ has the Hodge structure and the quadratic form $q_{X}$.

Proposition 3.10. Let $X, Y$ be compact irreducible symplectic Kähler manifolds. Assume $X$ is bimeromorphic to $Y$, i.e., there are proper modifications $f: Z \rightarrow X$ and $g: Z \rightarrow Y$ of $X$ and $Y$ respectively. Then the bimeromorphic map $h:=f \circ g^{-1}: Y \cdots \rightarrow X$ induces an isomorphism

$$
h^{*}=g ! \circ f^{*}: H^{2}(X, \mathbb{Z}) \rightarrow H^{2}(Y, \mathbb{Z}),
$$

preserving the Hodge structure and the quadratic forms $q_{X}$ and $q_{Y}$.

Proof. By Hironaka's desingularization theory we may assume $Z$ is smooth. Let $E$ and $F$ be, respectively the exceptional divisors of $f$ and $g$. Then the canonical bundle $K_{Z}$ of $Z$ is written as $K_{Z}=f^{*} K_{X}+E$ or $g^{*} K_{Y}+F$, where $K_{X}$ and $K_{Y}$ are, respectively, the canonical bundles of $X$ and $Y$, which are both trivial. Thus we have $E=F$ and hence $h$ defines a biholomorphic map of $X-f(E)$ to $Y-g(E)$. Note that both $f(E)$ and $g(E)$ are of codimension $\geq 2$. By Lemma 3.11 below it follows that $h$ induces an isomorphism $H^{2}(X, \mathbb{Q}) \cong H^{2}(Y, \mathbb{Q})$. Let $\varphi$ be a holomorphic symplectic form on $X$. Then $h^{*} \varphi$ extends holomorphically over the analytic set $g(E)$ of codimension $\geq 2$ and defines a symplectic form on $Y$. Since $h^{2,0}(X)=h^{2,0}(Y)=1$, this implies that $h^{*}$ preserves the Hodge structure. Also we have $h^{*} q_{X}=q_{Y}$ since the quadratic forms $q_{X}$ and $q_{Y}$ depends only on the symplectic structure $((2)$ of Theorem 3.9 ).

Q.E.D. 
Lemma 3.11. Let $M$ be a compact complex manifold of dimension $n$ and $V \subset M$ an analytic subset of codimension $\geq 2$. Then the inclusion map $M-V \hookrightarrow M$ induces an isomorphism $H^{2}(M, \mathbb{Z}) \cong H^{2}(M-V, \mathbb{Z})$. $V)$ :

Proof. Consider the cohomology exact sequence of a pair $(M, M-$

$$
H^{2}(M, M-V) \rightarrow H^{2}(M) \rightarrow H^{2}(M-V) \rightarrow H^{2}(M, M-V),
$$

where the coefficient $\mathbb{Z}$ is understood and omitted. $H^{i}(M, M-V) \cong$ $H_{2 n-i}(V)$ by the Alexander duality theorem; and $H_{2 n-i}(V)=0$ for $i=2$ or 3 since $\operatorname{dim}_{\mathbb{R}} V \leq 2 n-4$. Thus $H^{2}(M) \cong H^{2}(M-V)$. Q.E.D.

\section{$\S 4$. Period map and Weil-Peterson metric}

Let $M$ be a compact $n$-dimensional Kähler manifold with $c_{1}(M)_{\mathbb{R}}=$ 0 . Let $h^{r, 0}(M):=\operatorname{dim} H^{0}\left(M, \Omega^{r}\right)$. In this section we consider periods of holomorphic r-forms. According to the Bogomolov decomposition (see Theorem 1.1) the study of periods is reduced to the following three cases:

(1) $h^{n, 0}(M)=1, n=\operatorname{dim} M$, i.e., the canonical bundle of $M$ is trivial;

(2) $h^{2,0}(M)=1$, the case where $M$ is an irreducible symplectic Kähler manifold;

(3) the case where $M$ is a complex torus.

Starting with a general situation, we will later restrict our attention to the cases (1) and (2).

Polarized family. A pair $(M, \omega)$ of a compact Kähler manifold and a Kähler class $\omega \in H^{2}(M, \mathbb{R})$ is called a polarized Kähler manifold. Two polarized Kähler manifolds $(M, \omega)$ and $\left(M^{\prime}, \omega^{\prime}\right)$ are isomorphic if there is a biholomorphic map $f: M \rightarrow M^{\prime}$ with $f^{*} \omega^{\prime}=\omega$. A polarized deformation family $(\mathcal{M} \rightarrow T, \sigma)$ of a polarized Kähler manifold $(M, \omega)$ is a deformation family $\pi: \mathcal{M} \rightarrow T$ of $M$ with a section $\sigma \in \Gamma\left(S, R^{2} \pi_{*} \mathbb{R}\right)$ such that 1) $M \cong M_{o}, \omega=\sigma(o)$ for some $o \in T$; and 2) $\omega_{t}:=\sigma(t)$ is a Kähler class on $M_{t}$ for each $t \in T$. The universal polarized deformation family is defined analogously to the usual deformation. Existence of an universal polarized deformation family of any polarized Kähler manifold $(M, \omega)$ follows from the existence of the usual universal deformation family of $M$.

Theorem 4.1. The universal polarized deformation space of a polarized Kähler manifold $(M, \omega)$ with $c_{1}(M)_{\mathbb{B}}=0$ is smooth. 
Let $(M, \omega)$ be a polarized Kähler manifold with $c_{1}(M)_{\mathbb{R}}=0$. Let $(\pi: \mathcal{M} \rightarrow T, \sigma)$ be its universal polarized deformation family with $M \cong$ $\pi^{-1}(o), o \in T$. Then $T$ is, as a germ at $o$, an analytic subset of the universal deformation space $S$ of $M$. The tangent space of $S$ at $o$ is identified with $H^{1}(M, \Theta)$; the tangent space of $T$ at $o$ is the linear subspace

$$
\left.H^{1}(M, \Theta)_{\omega}:=\left\{\theta \in H^{1}(M, \Theta) \mid \theta\right\lrcorner \omega=0\right\}
$$

where the symbol $\downarrow$ means a product

$$
H^{1}(M, \Theta) \times H^{1}\left(M, \Omega^{1}\right) \rightarrow H^{2}(M, \mathcal{O})
$$

induced by the contraction $\Theta \times \Omega^{1} \rightarrow \mathcal{O}$.

We note here

Proposition 4.3. Let $(\mathcal{M} \rightarrow T, \sigma)$ be a universal polarized deformation family of a polarized Kähler manifold $(M, \omega)$ with $c_{1}(M)_{\mathbb{R}}=0$. Let $g_{t}$ be the (unique) Ricci-flat Kähler metric on $M_{t}$ whose cohomology class is $\sigma(t)$. Then there is a $C^{\infty} d$-closed form $\Phi$ on $\mathcal{M}$ such that the restriction of $\Phi$ to $M_{t}$ is the Kähler form of $g_{t}$ for each $t \in T$. In particular $g_{t}$ is $C^{\infty}$ in $t$.

Weil-Peterson metric. Let $\mathcal{M} \rightarrow T$ be the universal polarized deformation family of a polarized Kähler manifold $(M, \omega)$ with $c_{1}(M)_{\mathbb{R}}=0$. Then $T$ has a canonical metric called the Weil-Peterson metric, which is defined as follows. Let $g$ be the Ricci-flat Kähler metric whose Kähler class is $\omega$. The tangent space of $T$ at $o, M \cong M_{o}$, with $H^{1}(M, \Theta)_{\omega}$ defined in (4.2). Moreover we identify each element of $H^{1}(M, \Theta)_{\omega}$ with its harmonic representative relative to the metric $g$. The Weil-Peterson metric $g_{W P}$ on $T$ is defined at $o$ by

$$
g_{W P}\left(\theta, \theta^{\prime}\right):=\int_{M}\left\langle\theta, \theta^{\prime}\right\rangle d v_{g}
$$

where $\left\langle\theta, \theta^{\prime}\right\rangle$ is an inner product of the $\Theta$-valued harmonic $(0,1)$-form $\theta$, $\theta^{\prime} \in H^{1}(M, \Theta)_{\omega}$ relative to $g$ and $d v_{g}$ is the volume form of $g$.

Period maps. Let $\pi: \mathcal{M} \rightarrow S$ be a local universal deformation family of $M$ and $M_{s}:=\pi^{-1}(s)$ with $M_{o} \cong M$. By the unobstructedness theorem (Theorem 2.1 of Section 2) the base space $S$ is smooth. Let $\Psi: M \times S \rightarrow \mathcal{M}$ be a $C^{\infty}$-trivialization. Assume $d:=h^{r, 0}(M) \neq 0$. Then, since $M$ and hence $M_{s}, s \in S$, are Kähler, every holomorphic r-forms on $M_{s}$ is $d$-closed, i.e., $H^{0}\left(M_{s}, \Omega^{r}\right) \subset H^{r}\left(M_{s}, \mathbb{C}\right)$ canonically, 
and $h^{r, 0}\left(M_{s}\right)=d$ are constant. Let $G r_{d}(V)$ denote the Grassmannian consisting of $d$-dimensional subspaces of a vector space $V$. A period map $p_{r}: S \rightarrow G r_{d}\left(H^{r}(M, \mathbb{C})\right)$ for holomorphic r-forms is defined by

$$
p_{r}(s):=\Psi^{*} H^{0}\left(M_{s}, \Omega^{r}\right) \in G r_{d}\left(H^{r}(M \times\{s\}, \mathbb{C})\right)=G r_{d}\left(H^{r}(M, \mathbb{C})\right) .
$$

Then $p_{r}$ is holomorphic, as proved by Griffiths [Gr] (in a more general setting).

Theorem 4.4 (local Torelli). The period map $p_{r}$ for holomorphic $r$-forms of compact Kähler manifolds with $c_{1, \mathbb{R}}=0$ is locally injective.

Proof. We shall show that the differential $\left(p_{r}\right)_{*}$ of $p_{r}$ at $o \in S$ is injective. Let $\rho: T_{o} S \rightarrow H^{1}(M, \Theta)$ be the Kodaira-Spencer map. The unobstructed theorem (Theorem 2.1 in Section 2) says that this map is an isomorphism. In view of the Hodge decomposition of $H^{r}(M, \mathbb{C})$, the tangent space of $G r_{d}\left(H^{r}(M, \mathbb{C})\right)$ at $p_{r}(o)$ is identified with

$$
\operatorname{Hom}\left(H^{0}\left(M, \Omega^{r}\right), H^{1}\left(M, \Omega^{r-1}\right) \oplus \cdots \oplus H^{r}(M, \mathcal{O})\right) .
$$

Then $\left(p_{r}\right)_{*}(v), v \in T_{o} S$, is a map $\left.\rho(v)\right\lrcorner \bullet$ induced by the contraction $\Theta \otimes \Omega^{r} \rightarrow \Omega^{r-1}$.

Now let $M$ equip with a Ricci-flat Kähler metric. Let $\theta$ a $\Theta$-valued harmonic $(0,1)$-form on $M$ and $\varphi$ be a holomorphic $\mathrm{r}$-form. Then the $(r-1,1)$-form $\theta\lrcorner \varphi$ obtained by contraction with $\Theta$-component is also harmonic since $\varphi$ is parallel by Proposition 1.4 in Section 1. Thus the de Rham cohomology class of $\theta\lrcorner \varphi$ does not vanish whenever $\theta \neq 0$ and $\varphi \neq 0$. It follows that $\left(p_{r}\right)_{*}$ is injective.

Q.E.D.

Let $(\mathcal{M} \rightarrow T, \sigma)$ be a deformation family of polarized Kähler manifolds $\left(M_{t}, w_{t}\right), t \in T$. For $r<n:=\operatorname{dim}_{\mathbb{C}} M$ let

$$
H^{r}\left(M_{t}, \mathbb{R}\right)_{0}:=\operatorname{Ker}\left(L_{t}^{n-r+1}: H^{r}\left(M_{t}, \mathbb{R}\right) \rightarrow H^{2 n-r+2}\left(M_{t}, \mathbb{R}\right)\right),
$$

where $L_{t}$ is the multiplication by the cohomology class of $\omega_{t}$. Namely $H^{r}\left(M_{t}, \mathbb{R}\right)_{0}$ is the space of primitive cohomology classes of degree $r$ relative to the Kähler class $\omega_{t}$. Let $\Psi: M \times T \rightarrow \mathcal{M}$ be a $C^{\infty}$-trivialization of the family $\mathcal{M} \rightarrow T$. Then, identifying $M \times\{t\}$ with $M$ as usual, we have $\omega=\Psi^{*}\left(\omega_{t}\right)$ and hence $\Psi^{*} H^{r}\left(M_{t}, \mathbb{R}\right)_{0}=H^{r}(M, \mathbb{R})_{0}$ for each $t \in T$. Since holomorphic forms always define primitive classes, the period map $p_{r}$ for the polarized family takes its value in ${ } r_{d}\left(H^{r}(M, \mathbb{C})_{0}\right)$, $d=h^{r, 0}(M)$.

Period domains. From now on we assume that $r=2$ or $n=$ $\operatorname{dim} M$ and $h^{r, 0}(M)=1$. Then the image of the period map $p_{r}$ is 
contained in a certain subset of $\mathbb{P}\left(H^{r}(M, \mathbb{C})\right)=G r_{1}\left(H^{r}(M, \mathbb{C})\right)$, which is a bounded symmetric domain of type III and written in general as follows: Let $V$ be a vector space over $\mathbb{R}$ and $Q$ a nondegenerate bilinear form on $V$. Set $V_{\mathbb{C}}=V \otimes \mathbb{C}$ and

$$
D(V, Q)=\left\{\ell \in \mathbb{P}\left(V_{\mathbb{C}}\right) \mid Q(\varphi, \bar{\varphi})>0, Q(\varphi, \varphi)=0 \quad \text { for } \varphi \in \ell\right\} .
$$

The automorphism group $G$ of $D(V, Q)$ is induced by the linear transformation group of $V$ which preserves $Q$. Let $L$ be the tautological line bundle over $\mathbb{P}\left(V_{\mathbb{C}}\right)$. Then $Q$ induces a $G$-invariant hermitian metric $h_{Q}$ on $\left.L\right|_{D(V, Q)}$. The curvature $\operatorname{Ric}\left(h_{Q}\right)$ of $h_{Q}$ defines the $G$-invariant Kähler form $\sqrt{-1} \operatorname{Ric}\left(h_{Q}\right)$ on $D(V, Q)$.

Suppose $M$ is a symplectic manifold with $h^{2,0}(M)=1$. Let $q$ be the quadratic form on $H^{2}(M, \mathbb{R})$ introduced by Beauville [Bea-2] and Fujiki [Fu-3] (see $\S 3$ ) and set $D_{2}(M):=D\left(H^{2}(M, \mathbb{R}), q\right)$. Then any small deformation $M_{s}$ of $M$ is also a symplectic Kähler manifold with $h^{2,0}\left(M_{s}\right)=1$; the period map $p_{2}$ takes its image in $D_{2}(M)$. Moreover we have

Theorem 4.6 [Bea-2]. Let $M$ be a symplectic Kähler manifold with $h^{2}(M)=1$. Let $\mathcal{M} \rightarrow S$ be the local universal deformation of $M$. Then the period map $p_{2}: S \rightarrow D_{2}(M)$ is locally isomorphic.

Proof. The differential of $p_{2}$ is injective by Theorem 4.4. Therefore it suffices to show $\operatorname{dim} S=\operatorname{dim} D_{2}(M)$. By the unobstructedness theorem (Theorem 2.1), we have $\operatorname{dim} S=\operatorname{dim} H^{1}(M, \Theta)$. The interior product with the holomorphic symplectic form yields an isomorphism $\Theta \cong \Omega^{1}$ and hence $H^{1}(M, \Theta) \cong H^{1}\left(M, \Omega^{1}\right)$. Since $h^{2,0}(M)=1$, we have $h^{1,1}(M)=b_{2}(M)-2$. On the other hand $D_{2}(M)$ is an open subset of a hypersurface in $\mathbb{P}\left(H^{2}(M, \mathbb{C})\right)$ and hence $\operatorname{dim} D_{2}(M)=b_{2}(M)-2$. Consequently $\operatorname{dim} S=\operatorname{dim} D_{2}(M)$.

For a polarized symplectic Kähler manifold $M$ with $h^{2,0}(M)=1$, we set $D_{2}(M)_{0}:=D\left(H^{2}(M, \mathbb{R})_{0}, q\right)$. Note that $D_{2}(M)_{0}$ with the Kähler form $\sqrt{-1} \operatorname{Ric}\left(h_{q}\right)$ is isometric to $S O_{0}\left(2, b_{2}(M)-3\right) / S O\left(b_{2}(M)-3\right)$ with the invariant metric.

Theorem 4.7 (Schumacher [Sc]). Let $\mathcal{M} \rightarrow T$ be a local universal polarized deformation family of a symplectic Kähler manifolds $M$. Then $\omega_{W P}=p_{2}^{*} \sqrt{-1} \operatorname{Ric}\left(h_{q}\right)$, i.e., the period map $p_{2}: T \rightarrow D_{2}(M)_{0}$ is a local isometry, between the Weil-Peterson metric $g_{W P}$ on $T$ and the invariant metric on $D_{2}(M)_{0}$. 
For periods of holomorphic $n$-forms, we take $Q$ in (4.5) to be the intersection form $I$ on $H^{n}(M, \mathbb{R})$ and set $D_{n}(M)_{0}:=D\left(H^{n}(M, \mathbb{R})_{0}, I\right)$.

Theorem 4.8 (Tian [Ti]). Let $\mathcal{M} \rightarrow T$ be a local universal polarized deformation family a compact $n$-dimensional Kähler manifold $M$ with trivial canonical bundle. Then $\omega_{W P}=p_{n}^{*} \sqrt{-1} \operatorname{Ric}\left(h_{I}\right)$, i.e., the period map $p_{n}: T \rightarrow D_{n}(M)_{0}$ is an isometric immersion between the Weil-Peterson metric $g_{W P}$ on $T$ and the invariant metric on $D_{n}(M)_{0}$.

Proofs of Theorems 4.7 and 4.8 by Schumacher and Tian respectively go parallel; we only sketch here the proof of Theorem 4.8. Let $M$ be a compact Kähler manifold with trivial canonical bundle. Let $\left(M_{t}, \omega_{t}\right), t \in$ $T$, be a local universal polarized deformation family of compact Kähler manifolds $M_{t}$ with trivial canonical bundle. Let $\psi_{t}$ be a holomorphic $n$-form on $M_{t}$ which depends on $t$ holomorphically. Fix $o \in T$ and we write $(M, \omega)$ and $\psi$ for $\left(M_{o}, \omega_{o}\right)$ and $\psi_{o}$ respectively. Let $\Phi$ be the Kähler form of the Ricci-flat Kähler metric $g$ on $M$ whose cohomology class is $\omega$. Since the Ricci curvature vanishes identically, we have $\psi \wedge \bar{\psi}=a \Phi^{n}$ for some constant $a$. We identify $\theta \in H^{1}(M, \Theta)_{\omega}$ with the $\Theta$-valued harmonic $(0,1)$-form. Then, since $\Phi$ is parallel, $\theta\lrcorner \Phi=0$ as form. It follows by a direct calculation

$$
(\theta\lrcorner \psi) \wedge(\overline{\theta\lrcorner \psi})=-c_{n}\|\theta\|^{2} \Phi^{n},
$$

where $c_{n}$ is a positive constant depends only on $n$. Therefore

$$
\left.g_{W P}(\theta, \theta)=-\int_{M}(\theta\lrcorner \psi\right) \wedge(\overline{\theta\lrcorner \psi}) / \int_{M} \psi \wedge \bar{\psi} .
$$

We compute next $\sqrt{-1} p_{n}^{*} \operatorname{Ric}\left(h_{I}\right)$. Regarding $t$ as a local coordinate of the local deformation space at $o$, we assume $\theta$ is the image of $\partial / \partial t$ by the Kodaira-Spencer map. Then

$$
\sqrt{-1} p_{n}^{*} \operatorname{Ric}\left(h_{I}\right)=-\left.\partial_{t} \partial_{\bar{t}} \log \int_{M} \psi_{t} \wedge \bar{\psi}_{t}\right|_{t=o} .
$$

We can take a local holomorphic coordinates $\left(z_{t}^{1}, \cdots, z_{t}^{n}\right)$ on $M_{t}$ depending on $t$ smoothly so that

$$
\theta=\left.\sum_{\alpha} \bar{\partial}\left(\frac{\partial z^{\alpha}}{\partial t}\right) \frac{\partial}{\partial z_{t}^{\alpha}}\right|_{t=o} .
$$

Using these coordinates we can compute

$$
\left.\xi:=\left.\frac{\partial \psi_{t}}{\partial t}\right|_{t=o}=\psi^{\prime}+\theta\right\lrcorner \psi_{o}
$$


where $\psi^{\prime}$ is a $(n, 0)$-form. Moreover the left hand side of the above is $d$-closed; $h\lrcorner \psi_{o}$ is also $d$-closed since $\psi_{o}$ is parallel and $\theta$ is harmonic. Therefore $\psi^{\prime}$ is a constant multiple of $\psi_{o}$. It follows

$$
\left.\left.I\left(\psi_{o}, \bar{\psi}_{o}\right) I(\xi, \bar{\xi})-I\left(v_{o}, \bar{\xi}\right) I\left(\xi, \bar{\psi}_{o}\right)=I\left(\psi_{o}, \bar{\psi}_{o}\right) I(\theta\lrcorner, \bar{\theta}\right\lrcorner \psi_{o}\right) .
$$

Combining these all together we obtain

$$
g_{W P}(\theta, \theta)=\sqrt{-1} p_{n}^{*}\left(\operatorname{Ric}\left(h_{I}\right)\right)(\theta, \bar{\theta}) .
$$

\section{$\S 5 . \quad$ Examples}

In this section we discuss constructions of compact irreducible symplectic Kähler manifolds. A 2-dimensional irreducible symplectic Kähler manifold is a K3 surface, which has a long history of study (cf. [BPV]). Recall that symplectic Kähler manifolds have even (complex) dimensions. Four dimensional examples were discovered by A. Fujiki. Generalizing Fujiki's construction, Beauville [Bea-2] gave two series of examples for each even dimension as follows.

The symplectic manifolds $S^{[r]}$. Let $S$ be a compact complex surface (i.e., complex 2-dimensional manifold). Let $\operatorname{Sym}^{r} S:=S^{r} / \mathfrak{S}_{r}$ be the $r$-th symmetric product of $S$, where the symmetric group $\mathfrak{S}_{r}$ acts on $S^{r}$ as permutaion of components. Let $\pi: S^{r} \rightarrow \operatorname{Sym}^{r} S$ be the quotient map. Let $\Delta$ be the set of points $\left(x_{1}, \cdots, x_{r}\right) \in S^{r}$ such that at least two components are equal; set $D:=\pi(\Delta)$. Then $\operatorname{Sym}^{r} S$, whose singular points set is $D$, has a desingularization $\epsilon: S^{[r]} \rightarrow \operatorname{Sym}^{r} S$ such that $E:=\epsilon^{-1}(D)$ is an irreducible divisor. (In fact $S^{[r]}$ is the Douady space which parametrizes all 0 -dimensional analytic subspaces $Z \subset S$ with $\lg \left(\mathcal{O}_{Z}\right)=r$. See [Fo-1], [Fo-2], [Ia] for details.) If $S$ is Kählerian, then so is $S^{[r]}$. In fact, if $S$ is Kählerian, then $\operatorname{Sym}^{r} S$ is a Kähler space according to Varachus [Va]. Moreover any monoidal transformation of a Kähler space is again a Kähler space by Campana [Cam].

Proposition 5.1. $S^{[r]}$ has a symplectic holomorphic 2-form provided that the canonical bundle of $S$ is trivial.

Proof. Let $\Delta_{3} \subset \Delta$ be the set points $\left(x_{1}, \cdots, x_{r}\right) \in S^{r}$ such that at least three components are equal. Set $S_{*}^{(r)}:=S^{(r)}-\pi\left(\Delta_{3}\right), D_{*}:=$ $D-\pi\left(\Delta_{3}\right)$ and $S_{*}^{[r]}:=S^{[r]}-\epsilon^{-1} \pi\left(\Delta_{3}\right)$. Then, since $E=\epsilon^{-1}(D)$ is irreducible, $\epsilon^{-1} \pi\left(\Delta_{3}\right)$ is of codimension 2 in $S^{[r]}$. Therefore it suffices to show that $S_{*}^{(r)}$ has a holomorphic symplectic 2 -form. 
The diagonal set $\Delta-\Delta_{3}$ is smooth of codimension 2 on $S_{*}^{r}$. Let $\mu: Q_{\Delta}\left(S_{*}^{r}\right) \rightarrow S_{*}^{r}$ be the monoidal transformation of $S_{*}^{r}$ along $\Delta-\Delta_{3}$. The action of $\mathfrak{S}_{r}$ extends to $Q_{\Delta}\left(S_{*}^{r}\right)$ and $S_{*}^{[r]}$ is identified with the quotient of $Q_{\Delta}\left(S_{*}^{r}\right)$ by $\mathfrak{S}_{r}$; let $\varpi: Q_{\Delta}\left(S_{*}^{r}\right) \rightarrow S_{*}^{[r]}$ be the quotient map. Thus we have a commutative diagram:

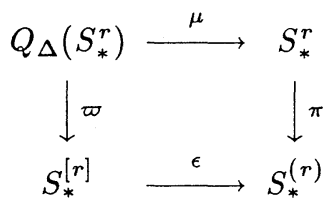

Let $\operatorname{pr}_{r}: S^{r} \rightarrow S$ be the projection to the $r$-th component and let $\varphi$ be a non-zero holomorphic 2 -form on $S$. Then $\psi_{0}:=\operatorname{pr}_{1}^{*} \varphi+\cdots+\operatorname{pr}_{r}^{*} \varphi$ is a symplectic 2 -form on $S^{r}$; and $\mu^{*} \psi_{0}$ is invariant under the action of $\mathfrak{S}_{r}$ on $Q_{\Delta}\left(S_{*}^{r}\right)$. The action of $\mathfrak{S}_{r}$ on $Q_{\Delta}\left(S_{*}^{r}-\Delta\right)$ is free from fixed points. If $g \in \mathfrak{S}_{r}$ fixes a point $p \in Q_{\Delta}\left(S_{*}^{r}\right)$, the tangent space of $Q_{\Delta}\left(S_{*}^{r}\right)$ at $p$ decomposes into a direct sum of $( \pm 1)$-eigen space; the (-1)-eigen space is one dimensional and the differential $\varpi_{*}$ of $\varpi$ is injective on the (+1)-eigen space. It follows that $\psi_{0}$ induces a holomorphic 2 -form $\psi$ on $S_{*}^{[r]}$ such that $\varpi^{*} \psi=\mu^{*} \psi_{0}$. The quotient map $\varpi$ is ramified along $E$ with local ramification index 2 . Therefore

$$
\operatorname{Zero}\left(\varpi^{*} \psi^{r}\right)=\varpi^{*} \operatorname{Zero}\left(\psi^{r}\right)+E,
$$

where Zero means a zero divisor with multiplicity. On the other hand, since $E$ is an exceptional divisor of $\mu$,

$$
\operatorname{Zero}\left(\varpi^{*} \psi^{r}\right)=\operatorname{Zero}\left(\mu^{*} \psi_{0}^{r}\right)+E .
$$

Thus $\psi^{r}$ vanishes nowhere.

Q.E.D.

There are two kinds of compact Kähler surfaces with trivial canonical bundle: K3 surfaces and complex 2-dimensional tori. For K3 surfaces we have

Proposition 5.2. Let $S$ be a K3 surface. Then $S^{[r]}$ is a simply connected irreducible symplectic Kähler manifold. There is an injective homomorphism $i: H^{2}(S, \mathbb{C}) \rightarrow H^{2}\left(S^{[r]}, \mathbb{C}\right)$, compatible to the Hodge structure, such that

$$
H^{2}\left(S^{[r]}, \mathbb{C}\right)=i\left(H^{2}(S, \mathbb{C})\right) \oplus \mathbb{C} \cdot[E] .
$$


For $\alpha \in H^{2}(S, \mathbb{C})$ we have $i(\alpha)=\mu^{*} \beta$, where $\beta \in H^{2}\left(S^{[r]}, \mathbb{C}\right)$ satisfies $\pi^{*} \beta=\sum_{i} \operatorname{pr}_{i}^{*} \alpha$ with the notation above.

The manifold $K m^{r}(T)$. Let $T$ be a 2-dimensional complex torus. Then $T^{[r+1]}$ is a symplectic Kähler manifold but it is not simply connected. In fact $\pi_{1}\left(T^{[r+1]}\right) \cong \pi_{1}(T)$; and the Albanese torus of $T^{[r+1]}$ is isomorphic to $T$ and the Albanese map $\alpha: T^{[r+1]} \rightarrow T$ is induced by the map $\left(x_{1}, \cdots, x_{r+1}\right) \in T^{r+1} \mapsto \sum_{i} x_{i} \in T$. Moreover, by the Bogomolov decomposition theorem (Theorem 1.1), $\alpha: T^{[r+1]} \rightarrow T$ is a holomorphic fiber bundle with structure group finite. Let $K m^{r}(T)$ denote the typical fiber of $\alpha$.

Proposition 5.3. $K m^{r}(T)$ is a simply connected irreducible symplectic Kähler manifold.

Deformations of $S^{[r]}$ and $K m^{r}(T)$. By deformation we have irreducible symplectic Kähler manifolds which are neither $S^{[r]}$ nor $K m^{r}(T)$. In fact Beauville [Bea-2] showed the following:

Theorem 5.4. Let $S$ be a K3 surface. Then the local universal deformation space $V$ of $S^{[r]}$ is of dimension 21. Each point of $V$ corresponds to an irreducible symplectic Kähler manifold; points corresponding to the manifolds of type $X^{[r]}$ with $X$ a K3 surface form a countable union of smooth hypersurfaces on $V$.

Theorem 5.5. Let $T$ be a complex torus of dimension 2. Then the local universal deformation space $V$ of $K \mathrm{~m}^{r}(T)$ is of dimension 5 . Each point of $V$ corresponds to an irreducible symplectic Kähler manifold; points corresponding to the manifolds of type $K m^{r}\left(T^{\prime}\right)$ with $T^{\prime}$ a 2-dimensional complex torus form a countable union of smooth hypersurfaces on $V$.

Elementary transformation. Although irreducible symplectic Kähler manifolds enjoy similar properties as K3 surfaces, there are phenomena peculiar to higher dimensional manifolds. For example Mukai [Mu-1] found

Theorem 5.6. Let $X$ be a symplectic manifold of dimension $2 n \geq$ 4 which contains a submanifold $Y$ isomorphic to the $n$-dimensional projective space $\mathbb{P}^{n}$. Then there are a symplectic manifold $X^{\vee}$ with a submanifold $Y^{\vee} \cong \mathbb{P}^{n}$ and a bimeromorphic map $f: X \cdots \rightarrow X^{\vee}$ such that $f$ does not define a holomorphic map on $Y$ but it induces a biholomorphic $\operatorname{map} X-Y \rightarrow X^{\vee}-Y^{\vee}$. 
$X^{\vee}$ is called an elementary transformation of $X$ along $Y$; and the construction goes as follows.

A $n$-dimensional complex submanifold $Y$ of a $2 n$-dimensional complex symplectic manifold $X$ with symplectic form $\varphi$ is called Lagrangean if $\iota_{Y}^{*} \varphi=0$ on $Y$, where $\iota_{Y}: Y \rightarrow X$ is the inclusion map. The bundle isomorphism $\varphi\left\llcorner: T X \cong T^{*} X\right.$ induces an isomorphism $T Y \cong N_{X / Y}^{*}$, where $N_{X / Y}$ is the normal bundle of $Y$ in $X$ and $N_{X / Y}^{*}$ is the dual bundle of $N_{X / Y}$. Assume now $Y \cong \mathbb{P}^{n}$. Let $\mu: X^{\square} \rightarrow X$ be the monoidal transformation of $X$ along $Y$ and let $Y^{\square}:=\mu^{-1}(Y)$. Then $Y^{\square}$ is isomorphic to the projectification $\mathbb{P}\left(N_{X / Y}\right)$ of $N_{X / Y}$. Since $N_{X / Y} \cong T^{*} Y$, it follows $Y^{\square} \cong \mathbb{P}\left(T^{*} Y\right)$. Let $Y^{\vee}$ denote the projective space dual to $Y=\mathbb{P}^{n}$, namely $Y$ parametrizes complex lines $\ell$ on $\mathbb{C}^{n+1}$ while $Y^{\vee}$ parametrizes hyperplanes. Then we have

$$
\mathbb{P}\left(T^{*} Y\right) \cong\left\{(\ell, H) \in Y \times Y^{\vee} \mid \ell \subset H\right\} .
$$

This means that $Y^{\square}$ admits a $P^{n-1}$-bundle structure $\nu: Y^{\square} \rightarrow Y^{\vee}$. Moreover we can blow down $X^{\square}$ onto a complex manifold $X^{\vee}$ along the fibers of $\nu$, namely $\nu$ extends to $X^{\square} \rightarrow X^{\vee}$ so that $\nu\left(Y^{\square}\right)=Y^{\vee}$. The elementary transformation $f$ is given by $\nu \circ \mu^{-1}$. Since $Y^{\vee}$ has codimension $n \geq 2$ in $Y^{\vee}$, the 2 -form $f^{*} \varphi$ on $X^{\vee}-Y^{\vee}$ extends to a holomorphic symplectic form on $Y^{\vee}$.

Counterexample to Torelli. For 2-dimensional compact irreducible symplectic Kähler manifolds, i.e., for K3 surfaces, the Torelli theorem holds:

Theorem 5.7 (Pjateckiī-Šapiro and Šafarevič [PS],[BR], [LP]). Two K3 surfaces $S, S^{\prime}$ are biholomorphic if and only if there is an isomorphism $h: H^{2}\left(S^{\prime}, \mathbb{Z}\right) \rightarrow H^{2}(S, \mathbb{Z})$ preserving the Hodge structure and the quadratic forms $q_{S^{\prime}}, q_{S}$.

For the higher dimensional case, however, this type of a theorem does not hold in a biholomorphic level. In fact Debarre [De] gave an example:

Proposition 5.8. There is a K3 surface $S$ such that

1) $X:=S^{[n]}$ admits an elementary transformation $h: X \cdots \rightarrow Y$;

2) $Y$ is an irreducible symplectic Kähler manifold not biholomorphic to $X$.

By Proposition $3.10, h$ induces an isomorphism $H^{2}(Y, \mathbb{Z}) \cong H^{2}(X, \mathbb{Z})$ which preserves the Hodge structure and the quadratic forms $q_{X}$ and $q_{Y}$. 


\section{References}

[Bca-1] Beauville, A., Surfaces K3, Sém.Bourbaki, 609 (1982/83).

[Bea-2] — Variete kähleriennes dont la premiere classe de Chern est nulle, J. Differential Geometry, 18 (1983), 755-782.

[Bea-3] — Some remarks on Kähler manifolds with $c_{1}=0$, in "Classification of Algebraic and Analytic Manifolds", Prog. Math. 39, Birkhäuser, 1983, pp. 71-250.

[Ber] Berger, M., Sur les groupes d'holonomie homogène des variétés a cnnexion affine et variétés riemanniennes, Bull. Soc. Math. France, 83 (1955), 279-330.

[Bo-1] Bogomolov, F., Kähler manifolds with trivial canonical class, Izv. Akad. Nauk SSSR. Ser. Mat., 38 (1974;). English transl. in Math. USSR. Izv. 8 (1974), 9-20

[Bo-2] On the decomposition of Kähler manifolds with trivial canonical class, Mat. Sb, 93 (1974). English transl. in Math. USSR-Sb. 22 (1974), 580-583

[Bo-3] — Hamiltonian Kähler manifolds, Dokl. Akad. Nauk SSSR, 19 (1978), 1426-1465. English transl. in Soviet Math. Dokl.

[Bo-4] Kähler varieties with trivial canonical class, preprint, 1981, I. H. E. S..

[BPV] Barth, W., Peters, C. and Van de Ven, A., "Compact complex surfaces", Springer-Verlag, 1984.

[BR] Burns, D. and Rapoport, M., On the Torelli problems for Kählerian K3 surfaces, Ann. Sci. École. Norm. Sup. 4th Ser., 8 (1975), 235-274.

[Ca-1] Calabi, E., On Kähler manifolds with vanishing canonical class, in "Algeraic Geometry and Topology", A symposium in honor of S. Lefschetz, Princeton University Press, 1957, pp. 78-89.

[Ca-2] - Isometric families of Kähler structures, in "The Chern Symposium, 1979", Springer-Verlag, 1980.

[CG] Cheeger, J. and D. Gromoll, The splitting theorem for manifolds of nonnegative Ricci curvature, J. Differential Geometry, 6 (1971), 119-128.

[De] Debarre, O, Un contre-exemple au théorème de Torelli pour la variétés symplectiques irréducibles, C. R. Acad. Sci Paris, 299 (1984), 681-684.

[Fo-1] Fogarty, J., Algebraic families on an algebraic surfaces, Amer. J. Math., 90 (1968), 511-521.

[Fo-2] - Algebraic families on an algebraic surface. II: the Picard scheme of the punctual Hilbert scheme, Amer. J. Math., 95 (1973), 660-687.

[Fu-1] Fujiki, A., On primitively symplectic compact Kähler V-manifolds of dimension four, in "Classification of Algebraic and Analytic Manifolds", Prog. Math. 39, Birkhäuser, 1983, pp. 71-250. 
[Fu-2] Coarse moduli space for polarized compact Kähler manifolds; Publ. RIMS, Kyoto Univ., 20 (1984), 977-1005.

[Fu-3] - On the cohomology group of a compact Kähler symplectic manifold, in "Algebraic Geometry, Sendai 1985,", Advanced Studies in Pure Math. 10, Kinokuniya and North-Holland, 1987, pp. 105-165.

[FW] Fisher, A. E. and J. A. Wolf, The structure of compact Ricci-flat Riemannian manifolds, J. Differential Geometry, 10 (1975), 277-288.

[Grr] Griffiths, P, Periods of integrals on algebraic manifolds, I, II, Amer. J. Math., 90 (1968), 568-626, 805-865.

[HW] Hirzebruch, F. and J. Werner, Some examples of threefolds with trivial canonical bundle, preprint, MPI, 1985, \# 58.

[Ia] Iarrobino, A, Punctual Hilbert schemes, Bull. Amer. Math. Soc., 78 (1972), 819-823.

[It] Itoh, M, Quaterion structure on the moduli space of Yang-Mills connections, Math. Ann., 276 (1986), 581-593.

[Ko-1] Kobayashi, S, Recent results in complex differential geometry, Jber. d. Dt. Math-Verein., 83 (1981), 147-158.

[Ko-2] L Simple vector bundles over symplectic Kähler manifolds, Proc. Japan Acad. Ser. A Math. Sci., 62 (1986), 21-24.

[Kod] Kodaira, K., "Complex manifolds and deformation of complex structures", Springer-Verlag, 1986.

[KB] Kobayashi, S. and Nomizu, K., "Foundation of differential geometry, I", Interscience, New York, 1963.

[KNS] Kodaira, K., Nirenberg, L. and Spencer, D. C., On the existence of deformations of complex analytic structures, Annals of Math., 68 (1958), 450-459.

[Koi] Koiso, N., Einstein metrics and complex structures, Invent. Math., 73 (1983), 41-106.

[Ku] Kuranishi, M., On the locally complete families of complex analytic structures, Annals of Math., 67 (1962), 536-577.

[Li-1] Lichenerowicz, A., Laplacien sur une variété riemannienne et spineur, Atti Accad. Naz Lincei Rend., 33 (1962), 187-191.

[Li-2] , "Global theory of connections and holonomy groups", Noordhoff, Leiden, 1976.

[LP] Looijenga, E., and Peters, C., Torelli theorems for Kähler K3 surfaces, Compositio Math., 42 (1981), 141-155.

[Ma] Matsushima, Y., Holomorphic vector fields and the first Chern class of a Hodge manifold, J. Differential Geometry, 3 (1969), 477-480.

[Mi-1] Michelson, M. L., Clifford and spinor cohomology of Kähler manifolds, Amer. J. Math., 102 (1980), 1083-1146.

[Mi-2] Kähler manifolds with vanishing first Chern class, in "Seminar on differential geometry", Ann. of Math. Stud. 102, Princeteton Univ. Press, 1982, pp. 359-361. 
[Mu-1] Mukai, S, Symplectic structure of the moduli space of sheaves on an abelian or K3 surface, Invent. Math., 77 (1984), 101-116.

[Mu-2] Moduli of vector bundles on K3 surfaces, and symplectic manifolds, Sūgaku, 39 (1987), 216-235;. English transl. in Sugaku Expositions 1\# 1, 1988

[Na] Nannicini, A., Weil-Petersson metric in the moduli space of compact polarized Kähler-Einstein manifolds of zero first Chern class, Manuscripta Math., 54 (1986), 405-438.

[PS] Pjateckiī-Šapiro, I. I., and Šafrevič, A Torelli theorem for algebraic surfaces of type K3, Izv. Akad. Nauk, SSSR, Ser. Mat., 35 (1971). English transl. 5(1971), 547-588

[Sc] Schumacher, G., On the geometry of moduli spaces, Manuscripta Math., 50 (1985), 229-267.

[Si] Siu, Y.-T., Every K3 surface is Kähler, Invent. Math., 73 (1983), 139-150.

[Si-2] Curvature of the Weil-Peterson metric on the moduli space of compact Kähler manifolds of negative Chern class, Aspect of Math. 9, Vieveg \& Sohn.

[Ti] Tian, G., Smoothness of the universal deformation space of compact Calabi-Yau manifolds and its Peterson-Weil metric, in "Mathematical Aspect of String Theory, 1986", Adv. Studies in Math. Physics 1, ed. S.-T. Yau,, World Scientific, 1987.

[To-1] Todorov, A. N., Moduli of hyper-Kählerian algebraic manifolds, preprint, MPI, 1985, no. 38 .

[To-2] The Weil-Peterson geometry of the moduli space of $\mathrm{SU}(n \geqq 3)$ (Calabi-Yau) manifolds I, preprint, MPI, 1987, no. 33.

[To-3] W Weil-Peterson geometry of Teichmüller space of Calabi-Yau manifolds (Torelli problem), preprint.

[Va] Varouchas, J, Stabilité de la classe des variétés Kähleriennes pour les certains morphisms propres, Invent. Math., 77 (1984), 117-128.

[Wa] Wakakuwa, H., On Riemannian manifolds with homogeneous holonomy group $S p(n)$, Tohoku Math. J., 10 (1958), 274-303.

[We] Weil, A, "Variétés kähleriennes", Hermann, Paris, 1971.

[Weyl] Weyl, H., "Classical groups", Princeton Univ. Press, 1946.

[Ya] Yau, S.T., On the Ricci curvature of a compact Kähler manifold and the complex Monge-Ampère equations, I, Comm. Pure Appl. Math, 31 (1978), 339-411.

\author{
Department of Mathematics \\ College of General Education \\ Osaka University \\ Toyonaka, 560 Osaka \\ Japan
}

